\title{
Research on Outfitting Virtual Assembly based on Ergonomics
}

\author{
Zuhua Jiang ${ }^{1 *}$, Junguo $\mathrm{Lu}^{2}$, Yongjun $\mathrm{Ji}^{1}$, and Hongyuan $\mathrm{Zhu}^{1}$ \\ ${ }^{1}$ Department of Industrial Engineering and Management, Shanghai Jiao Tong University, 800 \\ Dongchuan Road, Shanghai 200240, PR China \\ ${ }^{2}$ Research Institute of shipbuilding technology, Shanghai Waigaoqiao Shipbuilding Co. Ltd., 3001 \\ Zhouhai Road, Shanghai 200137, PR China
}

\begin{abstract}
To improve the present situation where the assembly work of ship outfitting depends on manual experiential operation, the thesis, taking outfitting assembly operation as the research object and optimal assembly path as the target, puts forward the simulation method and procedure of outfitting virtual assembly considering operational posture, operational load, reachability and visibility and other ergonomic factors, taking pipe assembly operation of ship section as the application example. The results show that the method can obtain better assembly operation path, avoid interference of workers' operation process and improve the efficiency of assembly operation.
\end{abstract}

\section{Introduction}

The outfitting work in shipbuilding is characterized by a large amount of work, wide work scope and intensive labor force, accounting for more than half of the total work of shipbuilding. In the process of outfitting, the assembly sequence of outfitting equipment is design according to the experience of designers, while the assembly trajectory depends on the experience of field operating persons, which results in that there are some problems, such as unreasonable operational posture of the field operating person, assembly interference and poor reachability and visibility, and that it costs too much time for the assembly of outfitting equipment to influence shipbuilding cycle in the process of practical assembly. Therefore, the research on the method and procedure of outfitting virtual assembly combined with ergonomics that can carry on the simulation analysis of manual assembly through the virtual environment and find the ergonomic problems that may appear in the process of outfitting assembly, can improve the feasibility of outfitting operations.

\section{Related works}

The core concept of outfitting virtual assembly on the basis of ergonomics is that by means of virtual simulation, the assembly sequence and assembly path of the fitting parts in the

\footnotetext{
${ }^{*}$ Corresponding author: Zhjiang@sjtu.edu.cn
} 
hull section are optimized to get the assembly path which is best suited to the workers in the field. Chaffin expounded that the digital human model has been increasingly used in industrial design system and ergonomic methods can evaluate the reachability of assembly design to reduce production cost and improve the comfort level of operation[1,2]. T. DUKIC et al proposed to add human model to the virtual assembly process, considering that the multifaceted ergonomic factors and methods can improve the authenticity of virtual simulation[3].Dan Lämkull et al. added virtual human into virtual assembly simulation, and by comparing with the actual operation result, concluded that digital human model can improve the operational posture and tooling design of the work, and can get better assembly sequence by forming a benign feedback process[4]. Shiguang Qiu et al.decomposed the virtual human movement into three kinds of movement, the original motion, fine motor and transient motion, realize the rapid generation of virtual people virtual human posture movement through hybrid control method of virtual human applied in the DMSP interactive assembly maintenance simulation system, which shows that the method can improve the generation efficiency of the virtual human movement[5].

For research on outfitting virtual assembly, most of the existing research focused on the single-site studies of assembly model building, design of assembly sequence, assembly path planning and the ergonomic evaluation methods without studying virtual assembly in different stages as a whole system. The optimization of assembly path is mainly focused on the optimization of the algorithm without optimizing the outfitting virtual assembly path in combination with ergonomic methods, which leads to poor practicability of optimized results. Focusing on above problems, this thesis, based on traditional outfitting virtual assembly methods and combined with ergonomic methods, puts forward the simulation method and procedure of outfitting virtual assembly considering operational posture, operational load, reachability and visibility and other ergonomic factors, constructs a systematic virtual assembly method and takes the pipe assembly operation of ship section as the application example.

\section{Outfitting virtual assembly method based on ergonomic analysis}

The virtual assembly processes based on ergonomics are shown in Figure 1. The virtual assembly process planning is a systematic work. Firstly, the design model is imported into the simulation system, and the initial assembly sequence is simulated and the assembly path is produced. The system can ensure the feasibility of outfitting assembly process by collision detection and constraint recognition. Combined with ergonomics, the outfitting assembly sequence and assembly process are optimized to ensure the reachability and visibility of assembly. Transfer the generated work files to the site, guide the field work, and feedback the work situation to the designers, so as to form the closed-loop feedback mechanism. The basic work processes are as follows.

(1) Designers firstly create a data model by CATIA, import the data model (including fittings equipment model, auxiliary tool model and model attribute information) model into the simulation software, DELMEL, and build the virtual assembly environment.

(2) the assembly designer shall, combined with the design experience, design the assembly sequence of the outfitting equipment according to the assembly process rules of the outfitting. According to the initial outfitting assembly sequence designed, control the assembly sequence of outfitting equipment and auxiliary tooling in virtual environment, set up the assembly sequence of outfitting parts, and generate the assembly path by the simulation system.

(3) In virtual assembly, simulation system can detect interference in virtual assembly through collision detection and constraint identification. If there is interference in the 
virtual assembly process, assembly designers will modify and optimize the assembly sequence and assembly path.

(4) If there is no interference, the virtual assembly process of the outfitting will be carried out ergonomic analysis, and the assembly sequence and assembly path of the outfitting shall be optimized.

(5) Finally, output the optimized assembly sequence and assembly path, generate the assembly process animation, the results of the assembly process planning, analysis results of assembly process and other process documents, transfer process documents to the field through the enterprise's integrated platform and provide guidance to on-site operating persons.

(6) Operating workers conduct actual assembly according to virtual assembly process documents provided, and feedback the problems existing in the actual assembly process through the enterprise integration platform to outfitting assembly designer to help them improve.

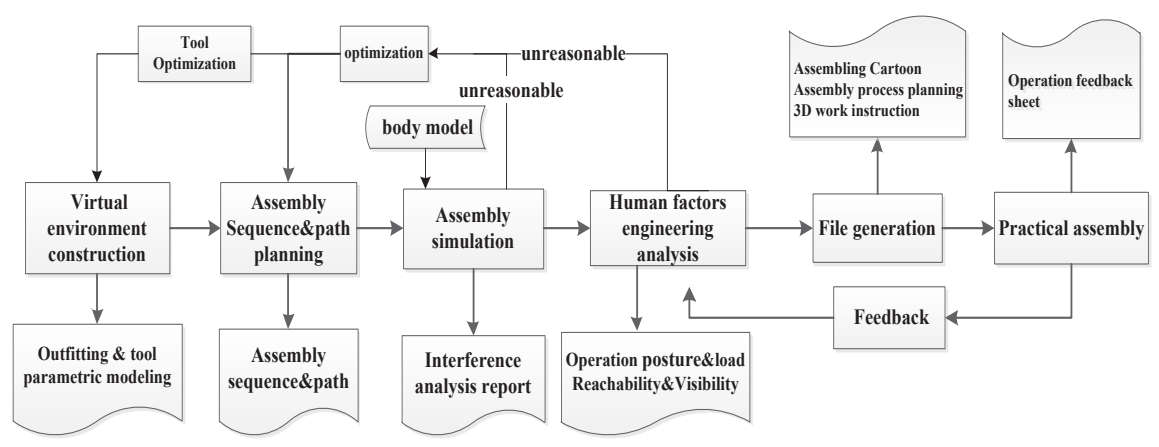

Fig. 1. Detailed process of outfitting virtual assembly based on ergonomic analysis

\section{Application example}

\subsection{Construction of virtual environment}

Taking the pipe virtual assembly of marine oil separator of a certain type as an example, build assembly scene, digital prototype 3D entity model, tool model and human model, and incorporate these models into the virtual environment. In order to build the model quickly, the model structure needs to be simplified, and some geometric information and constraint information should be removed. The virtual assembly maintenance environment created is shown in Figure 2.

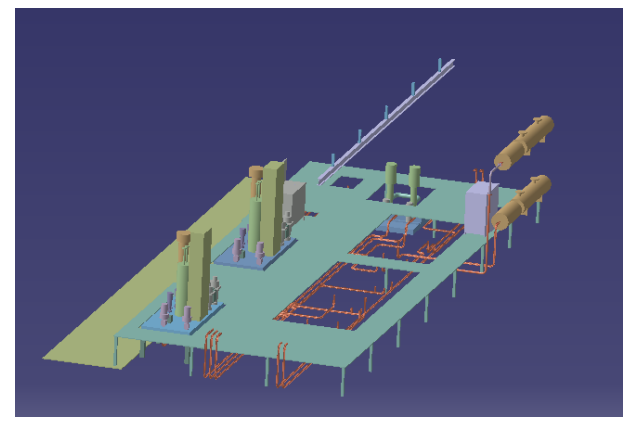

Fig. 2. The pipe virtual assembly environment of marine oil separator of a certain type 


\subsection{Virtual assembly path and simulation}

According to the path information of a piping system, establish the corresponding assembly path data file, generate the traditional assembly path in the DELMIA, and add the virtual human model, as shown in Figure 3.

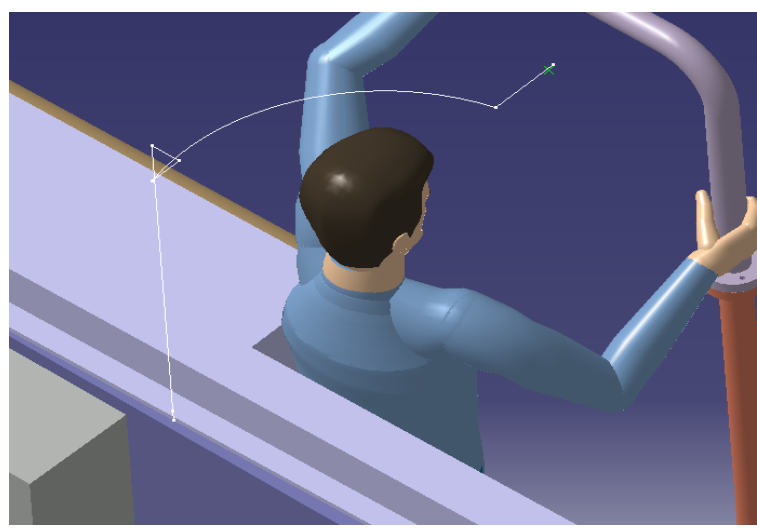

Fig. 3. Traditional assembly path

\subsection{Ergonomic analysis and optimization}

Because the size of the hull section is fixed and limited, the principle of global layout should be met in the installation of fittings and equipment. The general position of fittings and equipment has been determined, which limits the selection of people's installation posture. It is usually necessary to combine the activity space of the work, to analyze the traditional assembly path by ergonomic methods, and optimize the operational posture and operational load of the assembly to meet reachability and visibility.

\subsubsection{Ergonomic analysis}

Based on the traditional assembly path generated in DELMIA, according to the pipe assembly process, the simulation of virtual human operation can detect the situation of interference and invisibility in some sections of traditional assembly path generated previous, as shown in Figure 4.

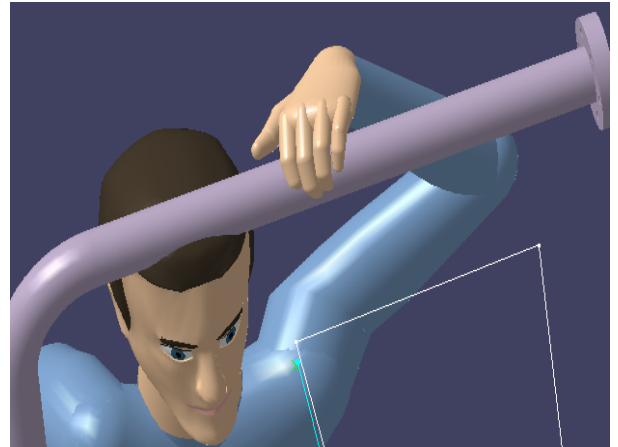

(a) Assembly interference

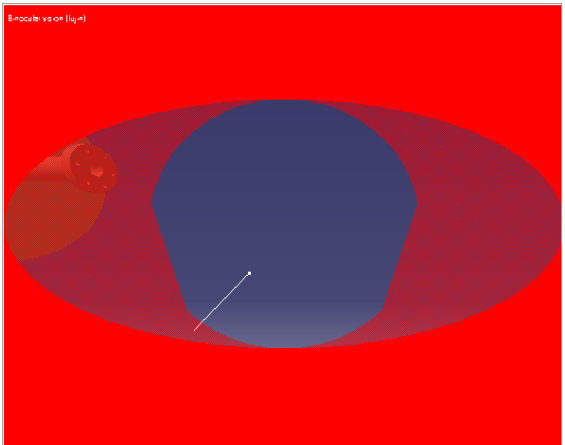

(b) Assembly invisibility

Fig. 4. Problems of traditional assembly path 


\subsubsection{Optimization of operational posture and load}

Using DELMIA RULA analysis method, grade the position comfort evaluation of virtual people, and give the load and the analysis scores of the various parts, to facilitate the optimization of operational posture and load. First analyze the traditional assembly path. As shown in figure 5, it can be seen under the scheduled posture, the right hand load of traditional assembly workers is bigger, and the left and right operational posture scored five points and three points, and even some of those joints up to 9 points, which is not conducive to workers in assembly operation and needs to be improved on the man-machine factors as soon as possible.

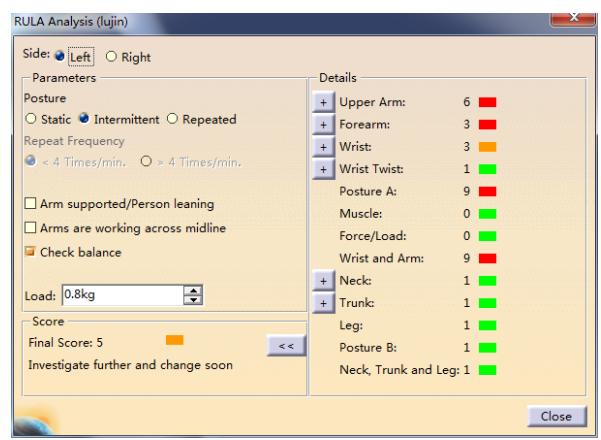

(a)Rula analysis of left limb

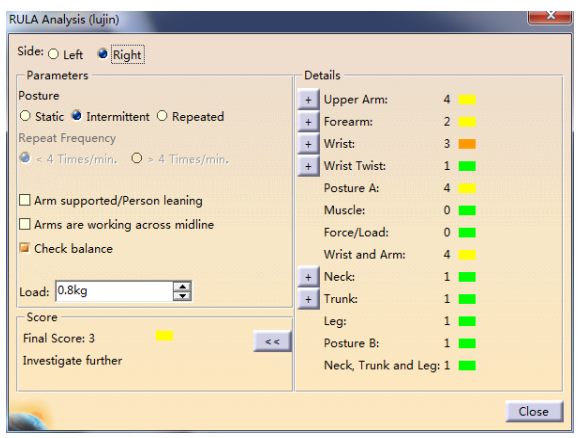

(b)Rula analysis of right limb

Fig. 5. Assembly posture of traditional assembly path and Rula analysis

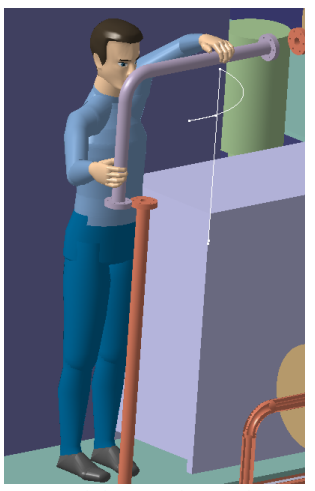

(a)Optimized assembly path and assembly posture

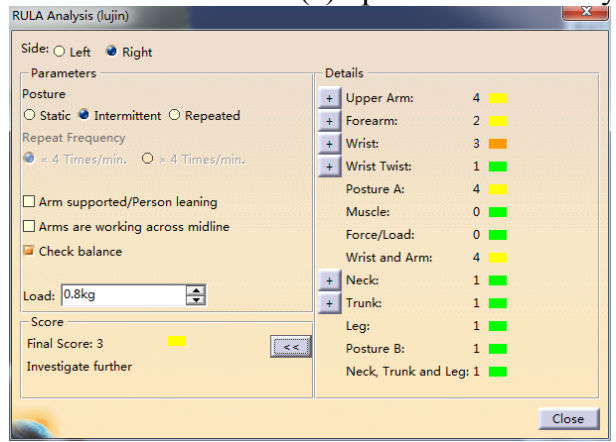

(b)Rula analysis of left limb

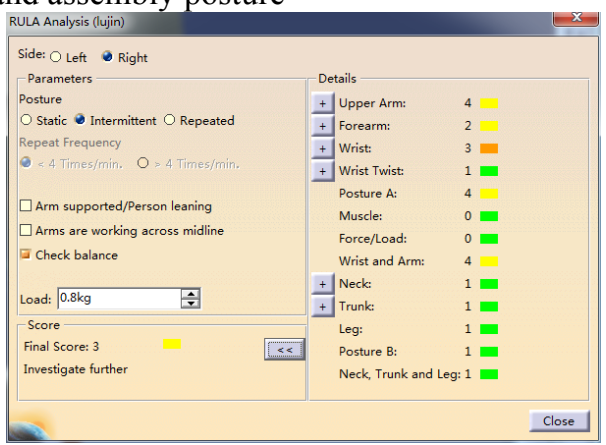

(c)Rula analysis of right limb

Fig. 6. Assembly posture and Rula analysis of new assembly path 
Combined with ergonomic factors, replan the pipe assembly path by DELMIA so that the operating load on the left and right side is relatively balanced during the assembly operation, and the operating posture scored 5 points and 3 points, as shown in Figure 6 . The suggestion is to further investigate. However, due to the influence of special space and part shape, the staff needs to adopt the uncomfortable posture to finish the work. But since the staff should hold the same position for a long time, this position is allowed.

\subsubsection{Analysis and optimization of reachability and visibility}

When workers carry out assembly operation at the installation site, the motion range of the hands and the vision at the posture are directly related to the feasibility of the assembly operation. Seen form Figure 7, the operating part is within the arm motion space and the operation reachability as well as visibility of pipe parts can be determined initially. But the location of the installation deviates from the center of the field of vision, that is, this is not the area with optimal field of vision, so it's necessary to adjust the orientation of the human model to find the best angle and position. The adjusted posture is shown in Figure 7. After the adjustment and the test, the operation reachability and the visibility are great.

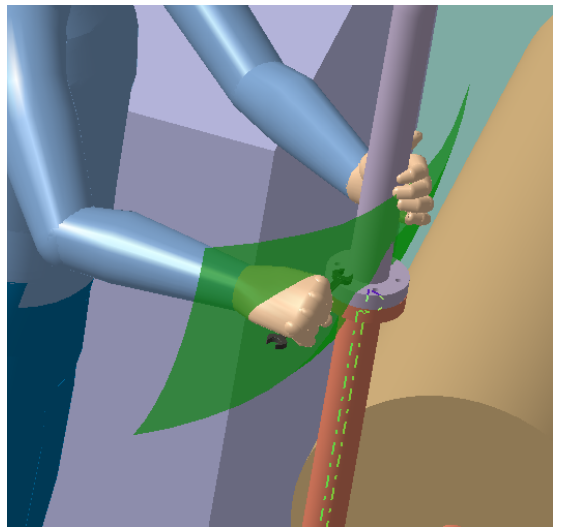

(a)Reach range before adjustment

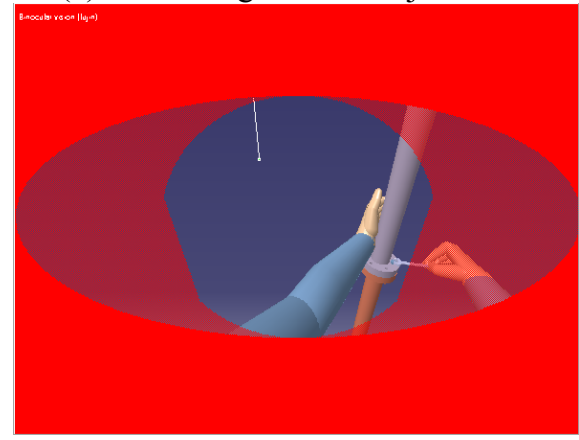

(c)Reach range before adjustment

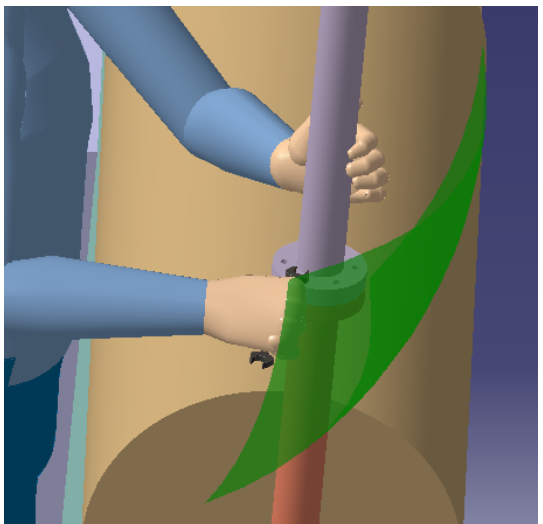

(b)Reach range after adjustment

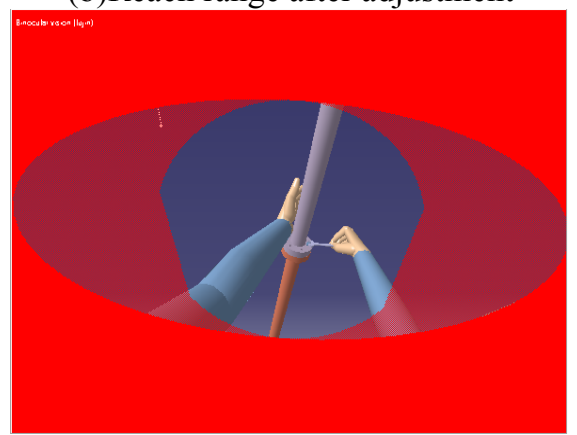

(d)Reach range after adjustment

Fig. 7. Reach range and visual range before adjustment

\section{Generation of Operation Instruction Documents and On-site Assessment}

Simulation and optimization can determine the assembly method that is to be used in practice. Transfer the virtual simulation documents of the final assembly scheme into the 
process documents that have the guiding effect on the process layout and operation of the actual assembly. Mainly include: making video data of virtual assembly process and matching with corresponding voice and subtitle, to form the guidance video of the pre-employment training; making an operation instruction book of document format printed in paper version, provided to the field operator.

On-site staff, based on the virtual simulation of the process document, carry out the construction and evaluation, feedback the results to the designer, forming a virtuous cycle of virtual assembly.

\section{Conclusion}

In this thesis, an outfitting virtual assembly method is put forward based on ergonomic analysis, and DELMEL software is used to optimize the pipe assembly path of the hull section. The study shows that in outfitting virtual simulation, the introduction of the man-machine engineering is able to optimize the workers' operational posture and operational load, test the assembly reachability and visibility and ensure the feasibility of outfitting assembly, so as to effectively guide the assembly of workers, ensure the quality of assembly and improve work efficiency.

This research was financially supported by Ministry of Industry and Information Technology of the People's Republic of China(The Ministry mounted Letter No.545[2016]).The authors gratefully acknowledge this support.

\section{References}

1. Chaffin D, On simulating human reach motions for ergonomics analyses, Human Factors and Ergonomics in Manufacturing, 12 (2002) 235-247

2. Chaffin D, Human motion simulation for vehicle and workplace design, Human Factors and Ergonomics in Manufacturing, 17 (2007) 475

3. T. Dukic, M. Rönnäng, M. Christmansson, Evaluation of ergonomics in a virtual manufacturing process, Journal of Engineering Design, 18 (2007), 125-137

4. Dan Lämkull, Lars Hanson, Roland örtengren, A comparative study of digital human modelling simulation results and their outcomes in reality: A case study within manual assembly of automobiles, International Journal of Industrial Ergonomics, 39 (2009) 428-441

5. Shiguang Qiu, Qichang He, Xiumin Fan \& Dianliang Wu, Virtual human hybrid control in virtual assembly and maintenance simulation, International Journal of Production Research, 52 (2014), 867-887 